\title{
Serological and molecular investigation of selected parasitic pathogens in European brown hare (Lepus europaeus) in Greece: inferring the ecological niche of Toxoplasma gondii and Leishmania infantum in hares
}

\author{
Constantina N. Tsokana ${ }^{1} \cdot$ Christos Sokos $^{1}$ - Alexios Giannakopoulos ${ }^{1}$ - Periklis Birtsas ${ }^{2,3} \cdot$ Labrini V. Athanasiou $^{4}$. \\ George Valiakos $^{1} \cdot$ Marina Sofia $^{1} \cdot$ Dimitrios C. Chatzopoulos ${ }^{1} \cdot$ Maria Kantere $^{1} \cdot$ Vassiliki Spyrou $^{5}$. \\ Angeliki Rodi Burriel ${ }^{6} \cdot$ Charalambos Billinis $^{1}$
}

Received: 4 October 2018 / Accepted: 27 June 2019 / Published online: 9 July 2019

(C) Springer-Verlag GmbH Germany, part of Springer Nature 2019

\begin{abstract}
The occurrence of infection or exposure to Toxoplasma gondii, Neospora caninum, and Leishmania infantum was investigated in European brown hares (Lepus europaeus, EBH) hunter-harvested over two consecutive hunting seasons in northern and central Greece. Geographical information system was used along with the ecological niche model to define the geographical distribution of seropositive hares relative to environmental parameters and to identify high-risk areas for hare exposure. Molecular analysis showed that $3.8 \%$ and $9.6 \%$ of the examined hares were infected with $N$. caninum and L. infantum, respectively, while, 5.7\%, $0.95 \%$, and $12.4 \%$ of the hares tested positive for the presence of antibodies against T. gondii, N. caninum, and L. infantum respectively. None of the examined hares was polymerase chain reaction-positive for $T$. gondii. Mixed exposure against both $T$. gondii and L. infantum was found in $2.9 \%$ of the hares examined. Rainfall indices and land uses significantly influenced the exposure of hares to T. gondii and L. infantum. This is the first molecular and serological survey of protozoan pathogens in EBH in Greece. Furthermore, we report the environmental parameters related to hare seropositivity and present a risk map for hare exposure to T. gondii and L. infantum in northern and central Greece. The ecological niches of T. gondii and L. infantum in the hares presented herein could be applied to other regions with similar environmental and climatic conditions.
\end{abstract}

Keywords GIS · Leishmania infantum · Lepus europaeus · MaxEnt · Neospora caninum · Toxoplasma gondii

Section Editor: Nawal Hijjawi

Charalambos Billinis

billinis@vet.uth.gr

1 Department of Microbiology and Parasitology, Faculty of Veterinary Medicine, University of Thessaly, Karditsa, Greece

2 Research Division, Hunting Federation of Macedonia and Thrace, Thessaloniki, Greece

3 Department of Forestry and Natural Environment Administration, Technological Institute of Thessaly, Karditsa, Greece

4 Department of Medicine, Faculty of Veterinary Medicine, University of Thessaly, Karditsa, Greece

5 Department of Animal Production, Technological Education Institute of Thessaly, Larissa, Greece

6 Department of Nursing, University of Peloponnesus, Sparti, Greece

\section{Introduction}

The epizootiological importance of the European brown hare (EBH) (Lepus europaeus) stems from the fact that it maintains the so-called home range, which makes it prone to many infectious diseases of natural nidality (Treml et al. 2007). Hares represent a potential source of Toxoplasma gondii and Neospora caninum for other animals, especially carnivores (Bártová et al. 2010). Recently, their role as a sylvatic reservoir of Leishmania infantum has been revealed as well (Tsokana et al. 2015).

Few epidemiological studies on wildlife have been conducted in Greece, and limited data are available on the parasitic pathogens in the hare population. This study aimed to (a) define the infection and exposure status of EBH from northern and central Greece against $T$. gondii, $N$. caninum, and $L$. infantum; (b) identify the environmental parameters related 
to the exposure of hares to $T$. gondii and $L$. infantum through geographical information system (GIS) and ecological niche model (ENM) analysis; and (c) create risk maps and define high-risk areas for the exposure of hares to these pathogens.

\section{Materials and methods}

\section{Hare samples, serological and molecular investigation, and sequencing}

From a total of 105 free-range hares, 105 serum samples and 52 liver samples were collected by hunters and foresters over two consecutive hunting seasons in northern and central Greece. Gender data of the animals included in the study were recorded when possible. The location data of the hares were marked with handheld global positioning system units (Garmin International, INC, Olathe, Kansas, USA) in the field.

The serum samples were tested with indirect fluorescence antibody test kits to detect antibodies against $T$. gondii, $N$. caninum (Agrolabo, Scarmagno, Italy), and L. infantum (Fuller Laboratories, Fullerton, CA, USA) by using a fluorescein-isothiocyanate-conjugated sheep anti-rabbit immunoglobulin G (BioFX Laboratories, Owings Mills, Maryland, USA).

Liver samples were subjected to polymerase chain reaction (PCR) tests to detect $T$. gondii (Homan et al. 2000), $N$. caninum (Müller et al. 1996), and L. infantum DNA (El Tai et al. 2001). Positive PCR products were purified using the PureLink PCR purification kit (Invitrogen, Carlsbad, CA, USA) and were bidirectionally sequenced using the fluorescent BigDye Terminator Cycle sequencing kit v3.1 (Applied Biosystems, Foster City, CA, USA), followed by fragment separation with a 3730xl DNA analyzer (Applied Biosystems, Foster City, CA, USA).

\section{Geographical information system and environmental parameters}

Climatic variables were sourced from the WorldClim website version 1.4. Wind speed was retrieved from GeoPortal (http:// www.rae.gr/geo/). Land uses, altitude, hydrological data, and the normalized difference vegetation index were sourced from the Copernicus program (https://land.copernicus.eu). All data layers were converted to a common projection, map extent, and resolution. ArcGIS 10.1 GIS software (ESRI, Redlands, CA, USA) was used for data analysis.

\section{Ecological niche model}

For ENM or species-distribution modeling programs, for example, MaxEnt (Phillips et al. 2006), a niche was defined based on the environmental values that correspond to "presence" data (in this case, T. gondii and L. infantum seropositive hares). Then, the similarities between environmental values at a specific cell and those of the niche of T. gondii and $L$. infantum seropositive hares were calculated for each raster cell in the study area to define the probability of occurrence of $T$. gondii and L. infantum seropositive hares in each raster cell.

In MaxEnt modeling (MaxEnt, software version 3.3.3), $T$. gondii and $L$. infantum seropositive hares were used as occurrence points in the ENM procedure. The goodness of fit of the model predictions was evaluated in terms of the mean area under the curve (AUC) of the receiver operating characteristic curve. We used the jackknife test until all remaining variables showed a positive effect on the total gain. Areas with $>63 \%$ probability of $T$. gondii and $L$. infantum seropositivity were considered high-risk areas.

\section{Statistical analysis}

The exact binomial test was performed to establish confidence intervals (CI) with a 95\% confidence level. Statistical analyses (chi-square and Fisher's exact tests to compare percentages of $T$. gondii and L. infantum seropositivity among the different study areas) were performed with IBM SPSS V.22.0 (IBM Corp, Armonk, New York, USA). A $p$ value $<0.05$ was considered statistically significant.

\section{Results and discussion}

Antibodies against T. gondii (5.7\%), N. caninum (0.95\%), and L. infantum (12.4\%) were detected in hares from central and northern Greece (Table 1, Fig. 1). The occurrence of $N$. caninum (3.8\%) and L. infantum (9.6\%) DNA and the lack of evidence for $T$. gondii infection were justified through molecular examination (Table 1). Sequence analyses of N. caninum and $L$. infantum amplicons (GenBank accession numbers MH568687 and KY379079-KY379080, respectively) revealed 96-98\% homology with other N. caninum sequences and 99\% homology with $L$. infantum. None of the $T$. gondii and $L$. infantum seropositive hares was found to be PCR positive (Table 1). No statistically significant difference was found in terms of seroprevalence for T. gondii $(p=0.45)$ and $L$. infantum ( $p=0.85$ ), as well as in terms of the prevalence of $L$. infantum DNA in Thessaly, central Macedonia, and eastern Macedonia and Thrace $(p=0.31)$ (Table 1).

Seroprevalence of $T$. gondii in hares varies among European countries, ranging from 0 to $46 \%$ (Poli et al. 1987; Aubert et al. 2010). Our findings (5.7\%) are consistent with those of studies conducted in Italy (6.4\%) (Poli et al. 1987) and France (9\%) (Aubert et al. 2010), as well as the seroprevalence reported in Greek wild boar (5.2\%) (Touloudi et al. 2015). Similar to the results of a study on the Iberian hare, $T$. gondii DNA was not detected in the hare liver samples 


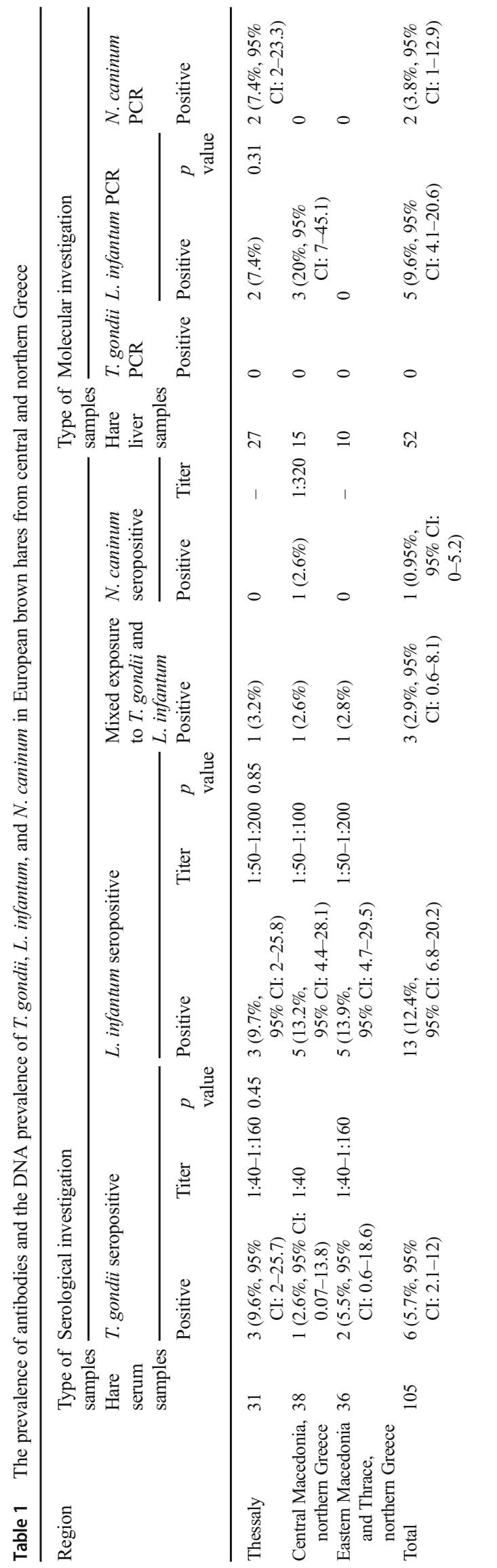

examined herein, possibly because of the presence of $T$. gondii DNA in other tissues, such as the brain or heart, as has been observed in subclinical infections in other animal species (Fernández-Aguilar et al. 2013).

The seroprevalence of $N$. caninum in hares ranges from 6.8 to 39\% (Ferroglio and Trisciuoglio 2003; Bártová et al. 2010), which is inconsistent with our findings; the low seroprevalence reported herein is comparable with the seroprevalence found in Greek wild boar (1.1\%) (Touloudi et al. 2015), indicating the existing although limited exposure of wildlife to $N$. caninum in the study area. Despite the low seroprevalence $(0.95 \%)$ and DNA prevalence $(3.8 \%)$ of $N$. caninum in this study, which could be perceived to be indicative of a low risk of infection through the consumption of infected hares in Greece, hares remain a constant source of carnivore infection. In Greece, when hares are hunted, the carcasses are eviscerated in the field, and the offal is purposely fed to hunting dogs or left behind for consumption by wild animals, including red foxes, a common predator of hares (Ferroglio and Trisciuoglio 2003). Although foxes have not been proved as a definitive host, their important role in sustaining $N$. caninum infection in the sylvatic cycle has been suggested based on their dietary habits and their adaptable and opportunistic nature that facilitates close contact with domestic dogs and livestock (Bateman and Fleming 2012).

A lower seroprevalence of $L$. infantum ( $0.9 \%$ compared with $12.4 \%$ in our study) was reported in L. europaeus in Italy (Ebani et al. 2016). Molecular studies have revealed 56.3\% L. infantum infection in Spain (Ruiz-Fons et al. 2013), and more recent studies have revealed $1.9 \%$ L. infantum infection in central Italy (Rocchigiani et al. 2018). In northern Greece, the $L$. infantum DNA prevalence reported for 2007-2011 was 23.49\% (Tsokana et al. 2015), which is consistent with our findings in the same region (20\%) (Table 1). The results of this study should be treated with caution given that a small subset of samples was molecularly examined to show the occurrence of infection rather than to determine the DNA prevalence of $L$. infantum infection in this hare population.

This study provides evidence of the occurrence of $L$. infantum infection in hares in central Greece as well, a highly endemic region for human and canine leishmaniasis, where an increase in the incidence rate of human leishmaniasis cases has been recorded in recent years (Giannakopoulos et al. 2016). In addition, it reports the seroprevalence of $L$. infantum in hares in central and northern Greece for the first time. The occurrence of $L$. infantum infection in hares, cats, dogs, rodents, red foxes, and humans (Diakou et al. 2009; Athanasiou et al. 2012; Ntais et al. 2013; Karayiannis et al. 2015; Giannakopoulos et al. 2016, 2017; Tsakmakidis et al. 2017) is highly suggestive of an overlapping of the sylvatic and the domestic transmission cycles in Thessaly, as has been suggested previously for the region of Thessaloniki, northern Greece (Tsokana et al. 2015). This hypothesis is further supported by the exposure of hares to 


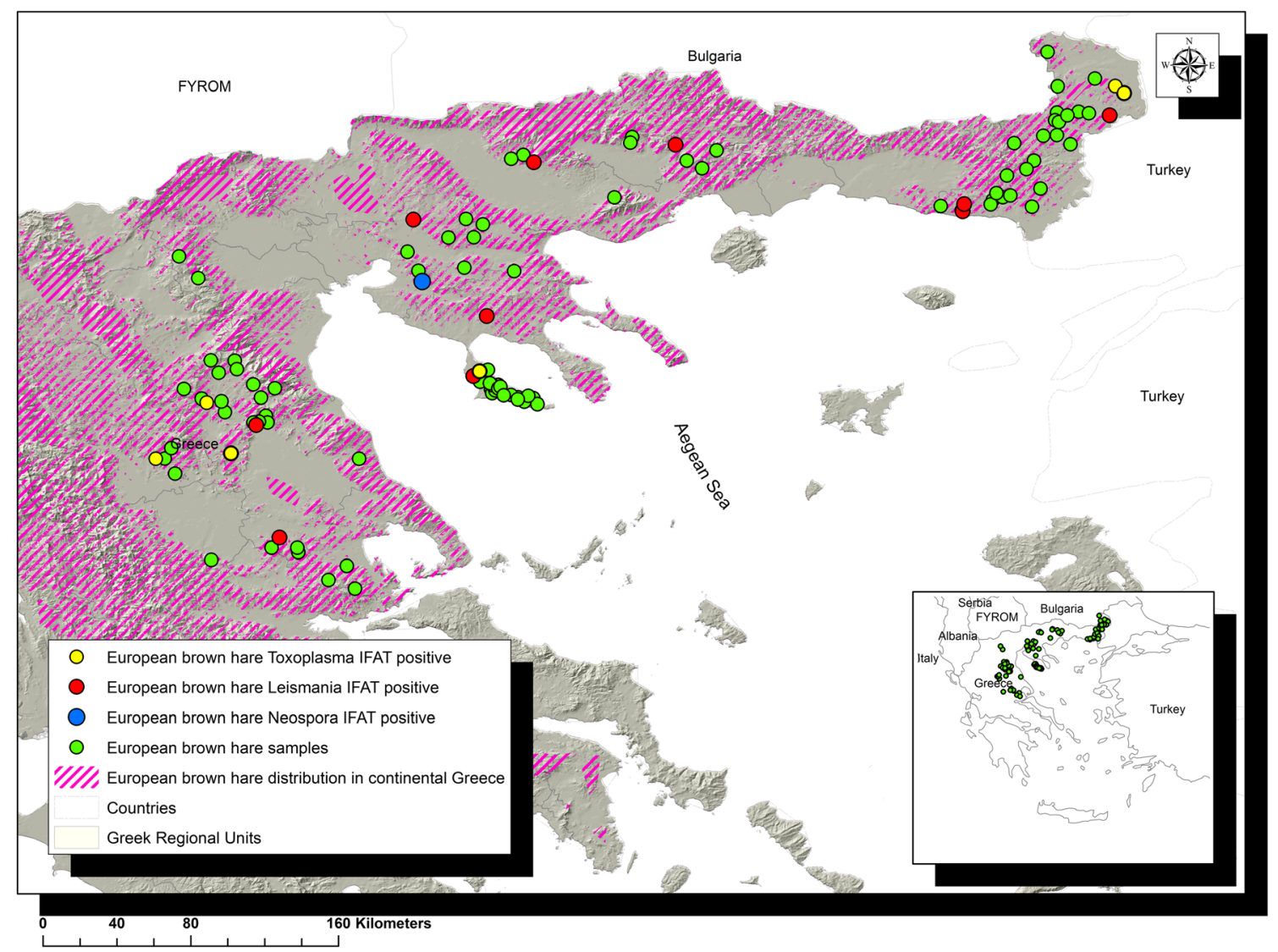

Fig. 1 Map of Greece showing the geographical distribution of the $T$. gondii, $N$. caninum, and L. infantum seropositive hares. Map of Greece showing the geographical distribution of the European Brown hare in continental Greece and the T. gondii, $N$. caninum, and L. infantum

$N$. caninum and $T$. gondii, indicating that hares share the same living space with the definitive hosts of these pathogens, namely, wild carnivores such as gray wolves and dogs, and cats or free-ranging felids respectively. Contact between hares and red foxes is well documented, as red foxes commonly prey on hares (Ferroglio and Trisciuoglio 2003). The fact that hares exposed to L. infantum share the same habitat with these animal species, which are susceptible to $L$. infantum infection (Millán et al. 2014), highlights the need to investigate the role of hares in the sylvatic transmission cycle of L. infantum in Greece. This is important when considering animal species such as cats; the behavioral characteristics of felines (proximity to humans; nocturnal predators operating in a $1.5-\mathrm{km}$ radius of their residence, using forests as hunting territory (Soares et al. 2016)), together with the so-called home range of hares, facilitate the cat-to-hare and the hare-to-cat spillovers of pathogens and cats an excellent candidate as a connective link between the sylvatic and the domestic transmission cycles of $L$. infantum spp. The same is true for red foxes because they dwell over great distances based on the availability of resources (Bateman and Fleming 2012).

The ENM from MaxEnt identified precipitation in the wettest month and land cover (habitat types) as the most influential seropositive hares found in central and northern Greece. Green dots indicate the hare samples collected in the study area. Yellow, blue, and red dots indicate the T. gondii, $N$. caninum, and L. infantum seropositive hares respectively

environmental variables associated with exposure of hares to T. gondii and L. infantum and identified high-risk areas for hare exposure through the creation of risk maps of the study areas (Fig. 2a, b). Precipitation influences the dynamics of T. gondii oocysts by creating a moist environment that facilitates their survival and increases food availability, thus supporting a high density of hosts, including transport hosts, such as arthropods, flies, cockroaches, and earthworms (Yan et al. 2016). Moreover, the prevalence of $T$. gondii has been associated with high levels of rainfall in studies involving other animal species, such as cats (Afonso et al. 2010). The habitat types associated with T. gondii seropositive hares (coniferous and mixed forests, non-irrigated arable land, and natural grasslands) may be linked to high population densities of important hosts, such as significant wildcat and wildcat-domestic hybrid populations in forests, resulting in increased soil contamination from their feces, high rodent density in arable lands owing to the availability of food, and the presence of transport hosts in natural grasslands owing to high soil permeability and rich organic matter. These habitats are characterized by moderate-to-high annual precipitation, which influences the dynamics of oocysts survival and dispersal. Soil irrigation and soil disturbance due to agricultural practices enhance oocyst survival and oocyst distribution in arable lands, 
Table 2 The relative contributions of the environmental variables to the Maxent model for the exposure of European brown hare to T. gondii and $L$. infantum

\begin{tabular}{|c|c|c|c|c|c|}
\hline \multicolumn{3}{|l|}{ T. gondii } & \multicolumn{3}{|l|}{ L. infantum } \\
\hline Variable & $\begin{array}{l}\text { Percent } \\
\text { contribution }\end{array}$ & $\begin{array}{l}\text { Permutation } \\
\text { importance }\end{array}$ & Variable & $\begin{array}{l}\text { Percent } \\
\text { contribution }\end{array}$ & $\begin{array}{l}\text { Permutation } \\
\text { importance }\end{array}$ \\
\hline Precipitation of wettest month & 27 & 6.2 & Precipitation of wettest month & 29.6 & 14.4 \\
\hline Corine land cover-habitat types & 24 & 22.2 & Corine land cover-habitat types & 21.3 & 12.7 \\
\hline Altitude & 18.9 & 16.4 & Altitude & 13 & 51.3 \\
\hline Livestock density & 12.1 & 14.5 & Livestock density & 9.2 & 0 \\
\hline Mean temperature of wettest quarter & 4.1 & 25.4 & Mean temperature of wettest quarter & 4.9 & 15.6 \\
\hline Farms distance & 0 & 0 & Farms distance & 0 & 0 \\
\hline Population density & 0 & 0 & Population density & 0 & 0 \\
\hline Wind speed & 0 & 0 & Wind speed & 1.6 & 1.7 \\
\hline $\begin{array}{l}\text { Normalized difference vegetation } \\
\text { index-September month }\end{array}$ & 0 & 0 & $\begin{array}{l}\text { Normalized difference vegetation } \\
\text { index-November month }\end{array}$ & 0 & 0 \\
\hline \multirow[t]{4}{*}{$\begin{array}{l}\text { Normalized difference vegetation } \\
\text { index-April month }\end{array}$} & 14 & 15.2 & $\begin{array}{l}\text { Normalized difference vegetation } \\
\text { index-April month }\end{array}$ & 0 & 0 \\
\hline & & & $\begin{array}{l}\text { Normalized difference vegetation } \\
\text { index-August month }\end{array}$ & 0 & 0.6 \\
\hline & & & Precipitation of wettest quarter & 0 & 0 \\
\hline & & & Min temperature of coldest month & 20.3 & 3.6 \\
\hline
\end{tabular}

while moist and shaded microenvironments facilitate the survival of oocysts in natural grasslands (Yan et al. 2016).

Regarding the exposure of hares to L. infantum, the impact of precipitation has been suggested in a study conducted among EBH in northern Greece (Tsokana et al. 2015). Precipitation affects the activity period of sandflies, their survival and the promotion of adult emergence, and the determination of appropriate oviposition sites (Quintana et al. 2013). The habitat types identified as being influential on hare exposure to L. infantum

a

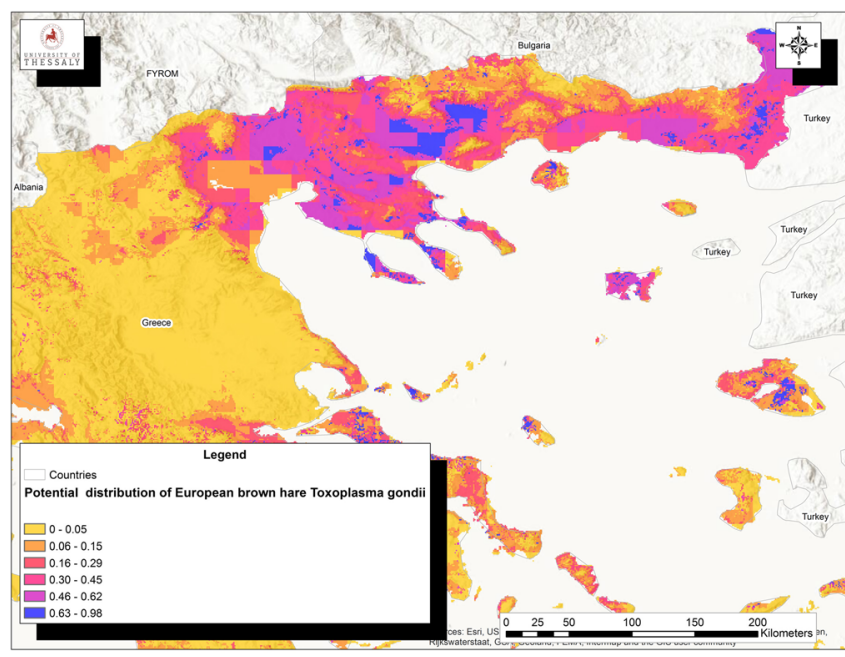

Fig. 2 Risk maps of Greece for the presence of $T$. gondii- and $L$. infantum-exposed hares. a Map of the study area showing the probability for the presence of $T$. gondii exposed hares together with the recognized high-risk areas for hare exposure to T. gondii. b Map of the (natural grasslands, coniferous forests, land principally occupied by agriculture, land with significant areas of natural vegetation, and non-irrigated arable land) are characterized by moderate-to-high annual precipitation, which influences the sandfly vector (Quintana et al. 2013). Furthermore, these types of habitats support a vast variety of mammals and diverse wildlife, including important hosts of $L$. infantum (e.g., free-ranging cats and rodents), owing to increased food availability, thus constituting an excellent space for the overlapping of the

\section{b}

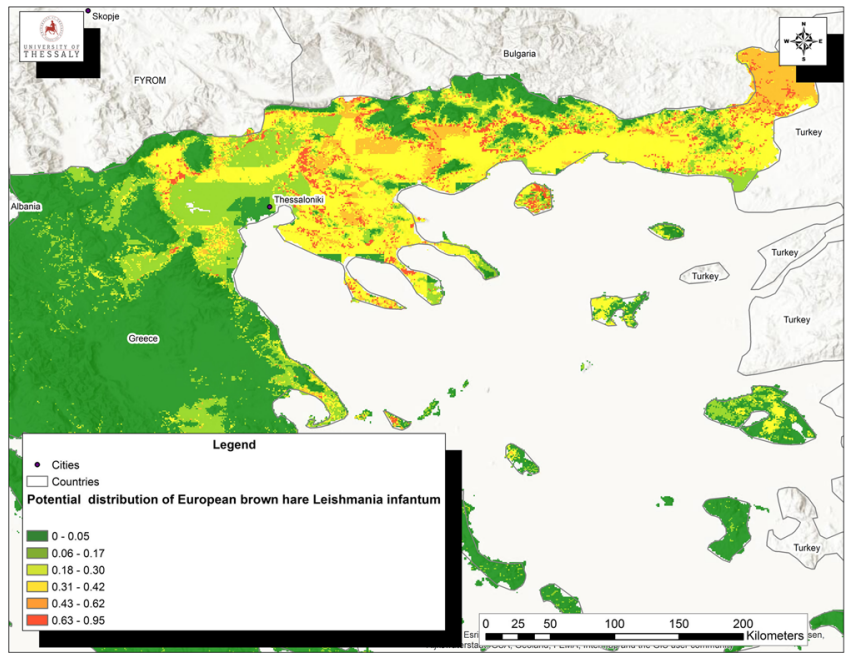

study area showing the probability for the presence of $L$. infantumexposed hares in the study area together with the recognized high-risk areas for hare exposure to L. infantum 
sylvatic and the domestic $L$. infantum transmission cycles. Agricultural and arable lands have been related to an increased probability of $L$. infantum infection in humans and dogs in Greece and other countries (Chamaillé et al. 2010; Ntais et al. 2013; Giannakopoulos et al. 2016).

Hares may significantly contribute to the epidemiology of important pathogens from the viewpoints of public health and veterinary concerns owing to cross-border and long-distance movements, possibly as part of live animal translocations; as hosts in intensive contact with vectors (ticks, mosquitoes, and sandflies); and as the prey of carnivores and omnivores that travel over great distances and come into contact with domestic animals and humans. Moreover, the short lifespan of hares makes them an excellent indicator of recent pathogen transmission and circulation in the area posing a risk of infection to wildlife, domestic animals, and humans.

Acknowledgments We thankfully acknowledge the foresters and hunters of the Hunting Federation of Macedonia and Thrace and the Hunting Federation of Thessaly and Sporades for providing the hare samples that were included in this study.

Funding information This research was co-financed by the European Union (European Social Fund-ESF) and national funds through the Operational Programme "Education and Lifelong Learning" of the National Strategic Reference Framework (NSRF)-Research Funded Project: THALES. Investing in the knowledge society through the European Social Fund (grant number MIS 377266).

\section{Compliance with ethical standards}

In this research, the authors did not perform any studies involving human participants or animals.

The hare serum and liver samples used in the study were collected opportunistically (no active capture, killing, and sampling of wild animals specifically for this study were performed) from animals hunter-harvested by members of the Greek Hunting Federation of Macedonia and Thrace and the Greek Hunting Federation of Thessaly and Sporades during the hunting seasons according to the prerequisites of the Greek Legislation and submitted to our laboratory for Passive Wildlife Disease Surveillance. Thus, special approval was not necessary, and steps to ameliorate suffering were not applicable to this study. Research on animals as defined in the EU Ethics for Researchers document (European Commission, 2007, Ethics for Researchers-Facilitating Research Excellence in FP7, Luxembourg: Office for Official Publications of the European Communities, ISBN 978-92-79-05474-7) is not applicable to this study.

Conflict of interest The authors declare that they have no conflict of interest.

\section{References}

Afonso E, Thulliez P, Gilot-Fromont E (2010) Local meteorological conditions, dynamics of seroconversion to Toxoplasma gondii in cats (Felis catus) and oocyst burden in a rural environment. Epidemiol Infect 138:1105-1113. https://doi.org/10.1017/ S0950268809991270
Athanasiou LV, Kontos VI, Saridomichelakis MN et al (2012) A crosssectional sero-epidemiological study of canine leishmaniasis in Greek mainland. Acta Trop 122:291-295. https://doi.org/10.1016/ j.actatropica.2012.02.003

Aubert D, Ajzenberg D, Richomme C et al (2010) Molecular and biological characteristics of Toxoplasma gondii isolates from wildlife in France. Vet Parasitol 171:346-349. https://doi.org/10.1016/j.vetpar. 2010.03.033

Bártová E, Sedlák K, Treml F et al (2010) Neospora caninum and Toxoplasma gondii antibodies in European brown hares in the Czech Republic, Slovakia and Austria. Vet Parasitol 171:155-158. https://doi.org/10.1016/j.vetpar.2010.03.002

Bateman PW, Fleming PA (2012) Big city life: carnivores in urban environments: urban carnivores. J Zool 287:1-23. https://doi.org/10. 1111/j.1469-7998.2011.00887.x

Chamaillé L, Tran A, Meunier A et al (2010) Environmental risk mapping of canine leishmaniasis in France. Parasit Vectors 3:31. https://doi. org/10.1186/1756-3305-3-31

Diakou A, Papadopoulos E, Lazarides K (2009) Specific anti-Leishmania spp. antibodies in stray cats in Greece. J Feline Med Surg 11:728730. https://doi.org/10.1016/j.jfms.2008.01.009

Ebani VV, Poli A, Rocchigiani G et al (2016) Serological survey on some pathogens in wild brown hares (Lepus europaeus) in Central Italy. Asian Pac J Trop Med 9:465-469. https://doi.org/10.1016/j.apjtm. 2016.03.032

El Tai NO, El Fari M, Mauricio I et al (2001) Leishmania donovani: intraspecific polymorphisms of Sudanese isolates revealed by PCR-based analyses and DNA sequencing. Exp Parasitol 97:3544. https://doi.org/10.1006/expr.2001.4592

Fernández-Aguilar X, Alzaga V, Villanúa D et al (2013) Epidemiology and prevalence of Toxoplasma gondii infection in the Iberian hare (Lepus granatensis). Vet Parasitol 196:194-198. https://doi.org/10. 1016/j.vetpar.2013.01.061

Ferroglio E, Trisciuoglio A (2003) Antibodies to Neospora caninum in European brown hare (Lepus europaeus). Vet Parasitol 115:75-78. https://doi.org/10.1016/S0304-4017(03)00201-2

Giannakopoulos A, Tsokana CN, Pervanidou D et al (2016) Environmental parameters as risk factors for human and canine Leishmania infection in Thessaly, Central Greece. Parasitology 143:1179-1186. https://doi.org/10.1017/S0031182016000378

Giannakopoulos A, Tsokana CN, Papadopoulos E et al (2017) Molecular investigation and geographical distribution of Leishmania spp. infection in stray and owned cats (Felis catus) in Thessaly, central Greece. J Hellenic Vet Med Soc 68:27-34

Homan W, Vercammen M, De Braekeleer J, Verschueren H (2000) Identification of a 200- to 300-fold repetitive 529 bp DNA fragment in Toxoplasma gondii, and its use for diagnostic and quantitative. Int J Parasitol 30:69-75. https://doi.org/10.1016/S0020-7519(99) 00170-8

Karayiannis S, Ntais P, Messaritakis I et al (2015) Detection of Leishmania infantum in red foxes (Vulpes vulpes) in Central Greece. Parasitology 142:1574-1578. https://doi.org/10.1017/ S0031182015001158

Millán J, Ferroglio E, Solano-Gallego L (2014) Role of wildlife in the epidemiology of Leishmania infantum infection in Europe. Parasitol Res 113:2005-2014. https://doi.org/10.1007/s00436-014-3929-2

Müller N, Zimmermann V, Hentrich B, Gottstein B (1996) Diagnosis of Neospora caninum and Toxoplasma gondii infection by PCR and DNA hybridization immunoassay. J Clin Microbiol 34:2850-2852

Ntais P, Sifaki-Pistola D, Christodoulou V et al (2013) Leishmaniases in Greece. Am J Trop Med Hyg 89:906-915. https://doi.org/10.4269/ ajtmh.13-0070

Phillips SJ, Anderson RP, Schapire RE (2006) Maximum entropy modeling of species geographic distributions. Ecol Model 190:231-259. https://doi.org/10.1016/j.ecolmodel.2005.03.026 
Poli A, Mancianti F, Marconcini A et al (1987) In: Ippen R, Schroder HD (eds) Diseases of wild-living hares (Lepus europaeus P.) in Tuscany. Akademie- Verlag, Berlin, pp 341-346

Quintana M, SalomóN O, Guerra R et al (2013) Phlebotominae of epidemiological importance in cutaneous leishmaniasis in northwestern Argentina: risk maps and ecological niche models. Med Vet Entomol 27:39-48. https://doi.org/10.1111/j.1365-2915.2012. 01033.x

Rocchigiani G, Ebani VV, Nardoni S et al (2018) Molecular survey on the occurrence of arthropod-borne pathogens in wild brown hares (Lepus europaeus) from Central Italy. Infect Genet Evol 59:142147. https://doi.org/10.1016/j.meegid.2018.02.005

Ruiz-Fons F, Ferroglio E, Gortázar C (2013) Leishmania infantum in free-ranging hares, Spain, 2004-2010. Euro Surveill Bull Eur Sur Mal Transm Eur Commun Dis Bull 18:20541

Soares CSA, Duarte SC, Sousa SR (2016) What do we know about feline leishmaniosis? J Feline Med Surg 18:435-442. https://doi.org/10. 1177/1098612X15589358

Touloudi A, Valiakos G, Athanasiou LV et al (2015) A serosurvey for selected pathogens in Greek European wild boar. Vet Rec Open 2: e000077. https://doi.org/10.1136/vetreco-2014-000077
Treml F, Pikula J, Bandouchova H, Horakova J (2007) European brown hare as a potential source of zoonotic agents. Vet Med (Praha) 52: $451-456$

Tsakmakidis I, Angelopoulou K, Dovas CI et al (2017) Leishmania infection in rodents in Greece. Trop Med Int Health TM IH 22:15231532. https://doi.org/10.1111/tmi.12982

Tsokana CN, Sokos C, Giannakopoulos A et al (2015) First evidence of Leishmania infection in European brown hare (Lepus europaeus) in Greece: GIS analysis and phylogenetic position within the Leishmania spp. Parasitol Res. https://doi.org/10.1007/s00436015-4749-8

Yan C, Liang L-J, Zheng K-Y, Zhu X-Q (2016) Impact of environmental factors on the emergence, transmission and distribution of Toxoplasma gondii. Parasit Vectors 9. https://doi.org/10.1186/ s13071-016-1432-6

Publisher's note Springer Nature remains neutral with regard to jurisdictional claims in published maps and institutional affiliations. 